\title{
Numerical solution of advection-diffusion equation using finite difference schemes
}

\author{
L. S. Andallah ${ }^{1}$ and M. R. Khatun ${ }^{2 *}$ \\ ${ }^{1}$ Department of Mathematics, Jahangirnagar University, Savar, Dhaka-1342, Bangladesh \\ ${ }^{2}$ Department of Mathematics, Bangabandhu Sheikh Mujibur Rahman Science and Technology University, Gopalgonj-8100
}

Received: 08 October 2018

Revised: 16 June 2019

Accepted: 01 July 2019

DOI: https://doi.org/10.3329/bjsir.v55i1.46728

\begin{abstract}
This paper presents numerical simulation of one-dimensional advection-diffusion equation. We study the analytical solution of advection diffusion equation as an initial value problem in infinite space and realize the qualitative behavior of the solution in terms of advection and diffusion co-efficient. We obtain the numerical solution of this equation by using explicit centered difference scheme and Crank-Nicolson scheme for prescribed initial and boundary data. We implement the numerical scheme by developing a computer programming code and present the stability analysis of Crank-Nicolson scheme for ADE. For the validity test, we perform error estimation of the numerical scheme and presented the numerical features of rate of convergence graphically. The qualitative behavior of the ADE for different choice of the advection and diffusion co-efficient is verified. Finally, we estimate the pollutant in a river at different times and different points by using these numerical scheme.
\end{abstract}

Keywords: Advection-Diffusion Equation; Explicit scheme; Crank-Nicolson scheme; Relative error; Rate of convergence

\section{Introduction}

Advection-diffusion equation is a parabolic partial differential equation which is derived on the principle of conservation of mass using Fick's 1st law. Particles, energy, or physical quantities are transferred inside a physical system due to two processes: advection and diffusion. Advection-diffusion equation describes this two process for several substances. The solution of this equation estimates some of the phenomena such as the contaminant transport in groundwater, spread of pollutants in rivers, contaminant dispersion in shallow lakes and reservoirs. It also considers physical phenomena where in the diffusion process particles are moving with certain velocity from higher concentration to lower concentration. The analytical and numerical solutions along with an initial condition and two boundary conditions aid to understand the pollutant concentration distribution behavior through an open medium like air, rivers, lakes and porous medium. River water pollution can be established by one-dimensional advection-diffusion equation. It has wide applications in other disciplines too, like soil physics, petroleum engineering, chemical engineering and biosciences. The finite difference method is one of the efficiently implementable numerical method and in this methodstability and convergence are important issue for obtaining results with good accuracy. To solve the advection-diffusion equation numerically, various work have been appeared by using the finite difference methods. An analytical solution of one dimensional advection-diffusion equation with variable coefficients in a finite domain using Laplace transformation technique. Researcher Tamora James (2002) made a numerical solution of ADE for Radial Flow. Romao et al. (2005) presented the finite difference methods of $3 \mathrm{D}$ convection diffusion equation to investigate error in the numerical solution of this equation. For this equation, Thongmoon and Mckibbin (2006) compared some numerical methods and indicated that FTCS and Crank-Nicolson scheme give 
better point-wise solutions than the spline methods. Agusta and Bamingbola (2007) studied on the numerical treatment of the mathematical model for water pollution. They used the implicit centered difference scheme in space and a forward difference method in time for the evaluation of the generalized transport equation. Changiun et al. (2010) made a numerical simulation on river water pollution by using grey differential model. They corrected the model in finding the truncation error and found that the obtained results from the grey model are better and logical. We estimate the relative error and the numerical features of the rate of convergence are presented graphically and also investigate the efficient numerical scheme for advection-diffusion equation. We examine the qualitative behavior of the solution of ADE under different parameters and examine mathematical models and following numerical methods to estimate the pollutants in a river at different times and different points using these numerical scheme.

\section{Materials and method}

\section{Analytic solution of Advection-Diffusion equation}

We consider the Advection-Diffusion equation as a Cauchy problem

$\frac{\partial c}{\partial t}+u \frac{\partial c}{\partial x}=D \frac{\partial^{2} c}{\partial x^{2}} ; t>0,-\infty<x<\infty$

With I.C $\mathrm{c}_{0}\left(t_{0}, x\right)=\mathrm{c}_{0}(x) ; \mathrm{a} \leq \mathrm{x} \leq \mathrm{b}$

By co-ordinate transformation, the analytic solution of advection-diffusion equation is given as

$$
c(x, t)=\frac{1}{\sqrt{4 \pi D t}} e^{(x-u t)^{2} / 4 D t}
$$

Which is the required solution of advection-diffusion equation.

\section{Numerical solution for Advection-Diffusion equation}

In order to implement the numerical scheme by finite difference method, we discretize the plane with mesh size. Grid width and time step are taken individually. The spatial and temporal coordinate at the grid point is defined as

$$
\begin{aligned}
& x_{i}=x_{0}+i \Delta x, \quad i=0,1,2, \ldots \ldots \ldots \ldots . . N \\
& t_{j}=t_{0}+j \Delta t, \quad j=0,1,2, \ldots \ldots \ldots \ldots . . T
\end{aligned}
$$

The approximate solution at a discrete set of points

$$
c\left(t^{n}, x_{i}\right) \approx c_{i}^{n} .
$$

Using Taylor's series expansion, we discretize the time derivative by forward difference formula

$$
\frac{\partial c\left(x_{i}^{n}\right)}{\partial t} \approx \frac{c_{i}^{n+1}-c_{i}^{n}}{\Delta t}
$$

The spatial derivative by the $1^{\text {st }}$ order backward difference formula

$$
\frac{\partial c\left(x_{i}^{n}\right)}{\partial x} \approx \frac{c_{i}^{n}-c_{i-1}^{n}}{\Delta x}
$$

The spatial derivative by the $2^{\text {nd }}$ order centered difference formula

$$
\frac{\partial c\left(x_{i}^{n}\right)}{\partial x} \approx \frac{c_{i+1}^{n}-c_{i-1}^{n}}{2 \Delta x}
$$

The spatial derivative by the $2^{\text {nd }}$ order centered difference formula

$\frac{\partial^{2} c\left(x_{i}^{n}\right)}{\partial x^{2}} \approx \frac{c_{i-1}^{n}-2 c_{i}^{n}+c_{i+1}^{n}}{\Delta x^{2}}$

\section{Explicit Centered Difference scheme}

Substituting equation (2), (3) and (5) into (1), we obtain

$c_{i}^{n+1}=(\alpha+\gamma) c_{i-1}^{n}+(1-\alpha-2 \gamma) c_{i}^{n}+\gamma c_{i+1}^{n}$

Which is known as explicit centered difference scheme by FTBSCS technique.

Again substituting equation (2), (4) and (5) into (1), we obtain

$c_{i}^{n+1}=\left(\frac{\alpha}{2}+\gamma\right) c_{i-1}^{n}+(1-2 \gamma) c_{i}^{n}+\left(\gamma-\frac{\alpha}{2}\right) c_{i+1}^{n}$

Which is known as explicit centered difference scheme by FTCSCS technique

where $\alpha=\frac{c \Delta t}{\Delta x}$ and $\gamma=\frac{D \Delta t}{\Delta x^{2}}$

Crank-Nicolson scheme

In numerical analysis the Crank Nicolson method is a finite difference method used for numerically solving the partial 
differential equation. The method was developed by John Crank and Phyllis Nicolson in the mid $20^{\text {th }}$ century for Diffusion equation.

Crank Nicolson scheme is a second order scheme which is obtained by the discretization of special and temporal derivative $\frac{\partial c}{\partial t}$ and $\frac{\partial^{2} c}{\partial x^{2}}$. Here we describe the discretization of the ADE analogous to Crank Nicolson scheme.

Now discretize the ADE at $\left(t+\frac{k}{2}\right) t h$ time step as $\frac{\partial c}{\partial t}\left(t+\frac{k}{2}\right)$ time step $+u \frac{\partial c}{\partial x}\left(t+\frac{k}{2}\right)$ time step $=D \frac{\partial^{2} c}{\partial x^{2}}\left(t+\frac{k}{2}\right)$ time step

$\Rightarrow \frac{\partial c}{\partial t}\left(t+\frac{k}{2}\right)$ th time step $+\frac{u}{2}\left[\frac{\partial c}{\partial x}(t)\right.$ th time step $+\frac{\partial c}{\partial x}(t+k)$ th time step $]=\frac{D}{2}\left[\frac{\partial^{2} c}{\partial x^{2}}(t)\right.$ th time step $+\frac{\partial^{2} c}{\partial x^{2}}(t+k)$ th time step]

Discretize the temporal derivative $\frac{\partial c}{\partial t}$ by central difference formula by Taylor's series expansion as

$c\left(t+\frac{k}{2}+\frac{k}{2}, x\right)=c\left(t+\frac{k}{2}, x\right)+\frac{k}{2} c_{t}\left(t+\frac{k}{2}, x\right)+$

$\frac{k^{2}}{4} \frac{1}{2 !} c_{t t}\left(t+\frac{k}{2}, x\right)+\ldots \ldots \ldots$

$c\left(t+\frac{k}{2}-\frac{k}{2}, x\right)=c\left(t+\frac{k}{2}, x\right)-\frac{k}{2} c_{t}\left(t+\frac{k}{2}, x\right)+$

$\frac{k^{2}}{4} \frac{1}{2 !} c_{t t}\left(t+\frac{k}{2}, x\right)-\ldots \ldots \ldots$

Substracting (9) and (10), we get,

$$
\begin{aligned}
& c(t+k, x)-c(t, x)=k c_{t}\left(t+\frac{k}{2}, x\right)+0\left(k^{3}\right) \\
& \Rightarrow c_{t}\left(t+\frac{k}{2}, x\right)=\frac{c(t+k, x)-c(t, x)}{k}+0\left(k^{2}\right) \\
& \Rightarrow \frac{\partial c}{\partial t}\left(t+\frac{k}{2}, x\right) \approx \frac{c(t+k, x)-c(t, x)}{k}
\end{aligned}
$$

Discretize the spatial derivative $\frac{\partial^{2} c}{\partial x^{2}}$ by centered difference formula. We obtain for $(t)$ th time step

$\frac{\partial c}{\partial x} \approx \frac{c(t, x+h)-c(t, x-h)}{h^{2}}$

And for $(t+k)$ th time step

$\frac{\partial c}{\partial x} \approx \frac{c(t+k, x+h)-c(t+k, x-h)}{h^{2}}$

Similarly, discretize the spatial derivative $\frac{\partial^{2} c}{\partial x^{2}}$ by centered difference formula for $(t)$ th time step

$\frac{\partial^{2} c}{\partial x^{2}} \approx \frac{c(t, x+h)-2 c(t, x)+c(t, x-h)}{h^{2}}$

And for $(t+k)$ th time step

$\frac{\partial^{2} c}{\partial x^{2}} \approx \frac{c(t+k, x+h)-2 c(t+k, x)+c(t+k, x-h)}{h^{2}}$

Putting this values in equation (8), we get

$$
\frac{c(t+k, x)-c(t, x)}{k}+
$$

$$
\begin{aligned}
& \frac{u}{2}\left[\begin{array}{l}
\frac{c(t, x+h)-c(t, x-h)}{h^{2}} \\
+\frac{c(t+k, x+h)-c(t+k, x-h)}{h^{2}}
\end{array}\right]= \\
& \frac{D}{2}\left[\begin{array}{l}
\frac{c(t, x+h)-2 c(t, x)+c(t, x-h)}{h^{2}}+ \\
\frac{c(t+k, x+h)-2 c(t+k, x)+c(t+k, x-h)}{h^{2}}
\end{array}\right]
\end{aligned}
$$

Now at a discrete set of points $\left(t^{n}, x_{i}\right)$

$$
\begin{aligned}
& \frac{c_{i}^{n+1}-c_{i}^{n}}{\Delta \mathrm{t}}+\frac{u}{2}\left(\frac{c_{i+1}^{n+1}-c_{i-1}^{n+1}}{2 \Delta x}+\frac{c_{i+1}^{n}-c_{i-1}^{n}}{2 \Delta x}\right)= \\
& \frac{D}{2}\left(\frac{c_{i-1}^{n+1}-2 c_{i}^{n+1}+c_{i+1}^{n+1}}{\Delta x^{2}}+\frac{c_{i-1}^{n}-2 c_{i}^{n}+c_{i+1}^{n}}{\Delta x^{2}}\right)
\end{aligned}
$$




$$
\begin{aligned}
& \Rightarrow c_{i}^{n+1}=c_{i}^{n}-\frac{u \Delta t}{4 \Delta x}\left(c_{i+1}^{n+1}-c_{i-1}^{n+1}+c_{i+1}^{n}-c_{i-1}^{n}\right)+ \\
& \frac{D \Delta t}{2 \Delta x^{2}}\left(c_{i-1}^{n+1}-2 c_{i}^{n+1}+c_{i+1}^{n+1}+c_{i-1}^{n}-2 c_{i}^{n}+c_{i+1}^{n}\right) \\
& \Rightarrow c_{i}^{n+1}+\frac{u \Delta t}{4 \Delta x}\left(c_{i+1}^{n+1}-c_{i-1}^{n+1}\right)-\frac{D \Delta t}{2 \Delta x^{2}}\left(\begin{array}{l}
c_{i-1}^{n+1}-2 c_{i}^{n+1} \\
+c_{i+1}^{n+1}
\end{array}\right)= \\
& c_{i}^{n}- \frac{u \Delta t}{4 \Delta x}\left(c_{i+1}^{n}-c_{i-1}^{n}\right)+\frac{D \Delta t}{2 \Delta x^{2}}\left(c_{i-1}^{n}-2 c_{i}^{n}+c_{i+1}^{n}\right) \\
& \Rightarrow\left(-\frac{\alpha}{4}-\frac{\gamma}{2}\right) c_{i-1}^{n+1}+(1+\gamma) c_{i}^{n+1}+\left(\frac{\alpha}{4}-\frac{\gamma}{2}\right) c_{i+1}^{n+1}= \\
&\left(\frac{\alpha}{4}+\frac{\gamma}{2}\right) c_{i-1}^{n}+(1-\gamma) c_{i}^{n}+\left(-\frac{\alpha}{4}+\frac{\gamma}{2}\right) c_{i+1}^{n}
\end{aligned}
$$

Which is known as Crank-Nicolson scheme and it is also known as CNS technique.

\section{Stability condition}

By the convex combination we obtain the stability condition of FTBSCS and FTCSCS. Equation (6) and (7) implies that the new solution is a convex combination of the two previous solutions. That is the solution at new time-step is an average of the solutions at the previous time-step at the spatial nodes. $i-1, \mathrm{i}$ and $i+1$.

The stability conditions of ADE by ECDS are as follow

$$
0 \leq \gamma \leq 1 \text { and }-\gamma \leq \alpha \leq 1-2 \gamma \text { (FTBSCS) }
$$

$0 \leq \alpha \leq 1$ and $0 \leq \gamma \leq \frac{1}{2}$ (FTCSCS)

Where $\alpha=\frac{u \Delta t}{\Delta x}$ and $\gamma=\frac{D \Delta t}{\Delta x^{2}}$

\section{Von neumann stability analysis for Crank-Vicolson scheme}

In numerical analysis, Von Neumann stability analysis is a procedure used to check the stability of finite difference scheme. The analysis was developed at Los Alamos National Laboratory after having been briefly described in a 1947 article by British researchers Crank and Nicolson. Later, the method was given a more rigorous treatment in an article co-authored by John Von Neumann. The Crank-Nicolson scheme of Advection-Diffusion equation is

$$
\begin{array}{r}
\left(-\frac{\alpha}{4}-\frac{\gamma}{2}\right) c_{j-1}^{n+1}+(1+\gamma) c_{j}^{n+1}+\left(\frac{\alpha}{4}-\frac{\gamma}{2}\right) c_{j+1}^{n+1}= \\
\left(\frac{\alpha}{4}+\frac{\gamma}{2}\right) c_{j-1}^{n}+(1-\gamma) c_{j}^{n}+\left(-\frac{\alpha}{4}+\frac{\gamma}{2}\right) c_{j+1}^{n}
\end{array}
$$

let $c_{j}^{n}=\xi^{n} e^{i k j \Delta x}$ into (16)

$$
\begin{aligned}
& \left(-\frac{\alpha}{4}-\frac{\gamma}{2}\right) \xi^{n+1} e^{i k(j-1) \Delta x}+(1+\gamma) \xi^{n+1} e^{i k j \Delta x}+ \\
& \left(\frac{\alpha}{4}-\frac{\gamma}{2}\right) \xi^{n+1} e^{i k(j+1) \Delta x}=\left(\frac{\alpha}{4}+\frac{\gamma}{2}\right) \xi^{n} e^{i k(j-1) \Delta x}+ \\
& (1-\gamma) \xi^{n} e^{i k j \Delta x}+\left(-\frac{\alpha}{4}+\frac{\gamma}{2}\right) \xi^{n} e^{i k(j+1) \Delta x} \\
& \Rightarrow \xi^{n+1} e^{i k j \Delta x}\left(\begin{array}{l}
1+\gamma+\frac{\alpha}{4}\left(e^{i k \Delta x}-e^{-i k \Delta x}\right)- \\
\frac{\gamma}{2}\left(e^{i k \Delta x}+e^{-i k \Delta x}\right)
\end{array}\right)= \\
& \xi^{n} e^{i k j \Delta x}\left(\begin{array}{l}
1-\gamma-\frac{\alpha}{4}\left(e^{i k \Delta x}-e^{-i k \Delta x}\right)+ \\
\frac{\gamma}{2}\left(e^{i k \Delta x}+e^{-i k \Delta x}\right)
\end{array}\right) \\
& \Rightarrow \xi^{n+1}\left(1+\frac{\alpha}{2} i \sin k \Delta x+2 \gamma \sin ^{2} \frac{k \Delta x}{2}\right)= \\
& \xi^{n}\left(1-\frac{\alpha}{2} i \sin k \Delta x-2 \gamma \sin ^{2} \frac{k \Delta x}{2}\right) \\
& \Rightarrow \xi^{n+1}=\frac{\left(1-\frac{\alpha}{2} i \sin k \Delta x-2 \gamma \sin ^{2} \frac{k \Delta x}{2}\right)}{\left(1+\frac{\alpha}{2} i \sin k \Delta x+2 \gamma \sin ^{2} \frac{k \Delta x}{2}\right)} \xi^{n}
\end{aligned}
$$

Now we find the amplification factor

$$
\begin{aligned}
\xi & =\frac{\left(1-2 \gamma \sin ^{2} \frac{k \Delta x}{2}+i\left(-\frac{\alpha}{2} \sin k \Delta x\right)\right)}{\left(1+2 \gamma \sin ^{2} \frac{k \Delta x}{2}+i\left(\frac{\alpha}{2} \sin k \Delta x\right)\right)} \\
\Rightarrow|\xi| & =\frac{\sqrt{\left(1-2 \gamma \sin ^{2} \frac{k \Delta x}{2}\right)^{2}+\left(-\frac{\alpha}{2} \sin k \Delta x\right)^{2}}}{\sqrt{\left(1+2 \gamma \sin ^{2} \frac{k \Delta x}{2}\right)^{2}+\left(\frac{\alpha}{2} \sin k \Delta x\right)^{2}}}
\end{aligned}
$$


$\Rightarrow|\xi|^{2}=\frac{\left(1-2 \gamma \sin ^{2} \frac{k \Delta x}{2}\right)^{2}+\left(-\frac{\alpha}{2} \sin k \Delta x\right)^{2}}{\left(1+2 \gamma \sin ^{2} \frac{k \Delta x}{2}\right)^{2}+\left(\frac{\alpha}{2} \sin k \Delta x\right)^{2}}$
$\Rightarrow|\xi|^{2}=\frac{\left(1-2 \gamma \sin ^{2} \frac{k \Delta x}{2}\right)^{2}+\frac{\alpha^{2}}{4} \sin ^{2} k \Delta x}{\left(1+2 \gamma \sin ^{2} \frac{k \Delta x}{2}\right)^{2}+\frac{\alpha^{2}}{4} \sin ^{2} k \Delta x}$

Since $\sin ^{2} \frac{k \Delta x}{2} \geq 0$ and $\alpha, \gamma>0$, it follows that $|\xi|<1$.

Consequently the crank-Nicolson method is unconditionally stable.

\section{Error estimation and convergence}

We compute the relative error between analytic solution and numerical solution for FTBSCS, FTCSCS and CNS to determine which scheme is best. Now, we compute the relative error in $\mathrm{L}_{1}$-norm defined by error $=\frac{\left\|c_{e}-c_{N}\right\|}{\left\|c_{e}\right\|}$ for all time where is $c_{e}$ and $c_{N}$ are the exact solution and numerical solution computed by finite difference scheme.

\section{Result and discussion}

\section{Relative error for FTBSCS, FTCSCS and CNS}

We present finite difference schemes for $u=0.3 \mathrm{~m} / \mathrm{s}$ and $D=.005 \mathrm{~m}^{2} / \mathrm{s}$ up to time second in temporal grid size in spatial domain $[0,50]$ with spatial grid size, which satisfy the stability condition.

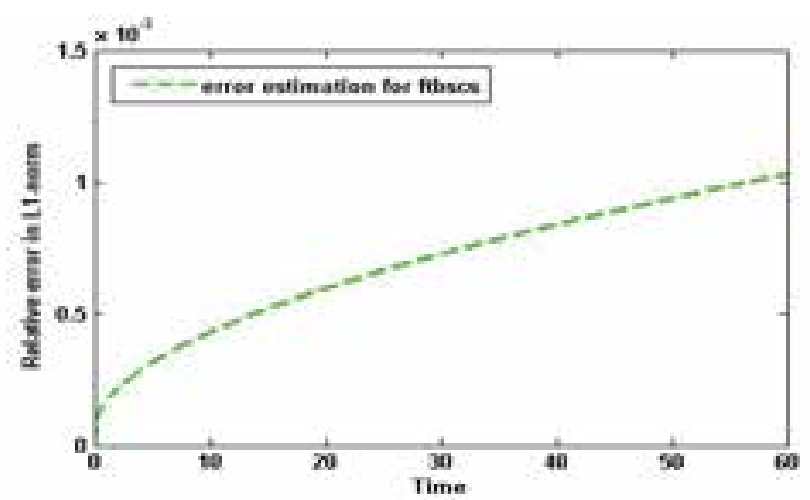

Fig. 1. Relative error of 1-D ADE for FTBSCS

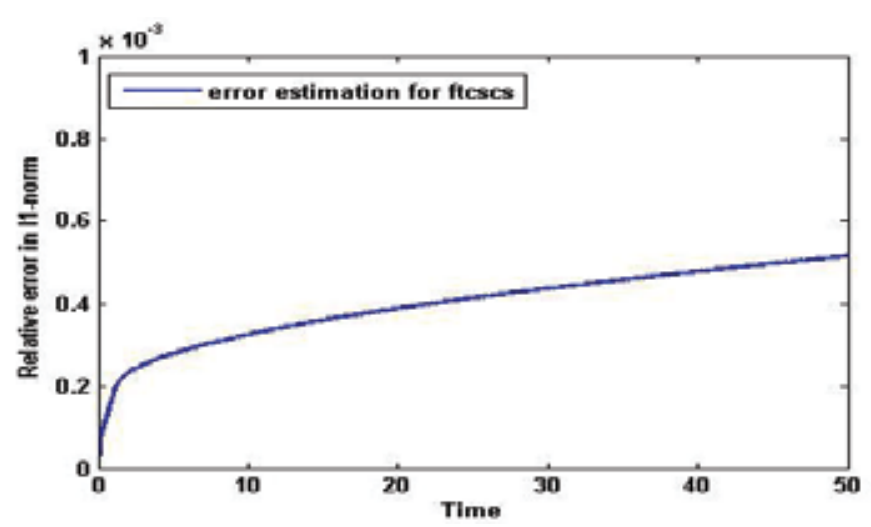

Fig. 2. Relative error of 1-D ADE for FTCSCS

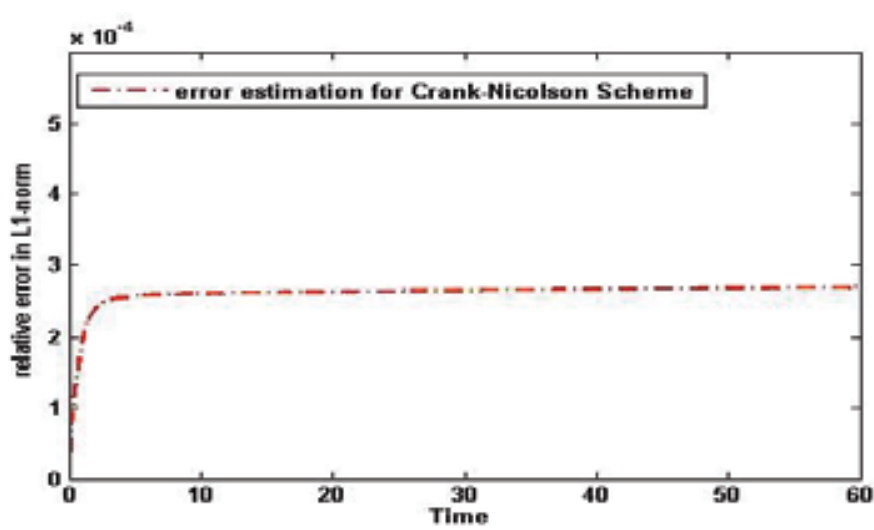

Fig. 3. Relative error of 1-D ADE for FTCSCS

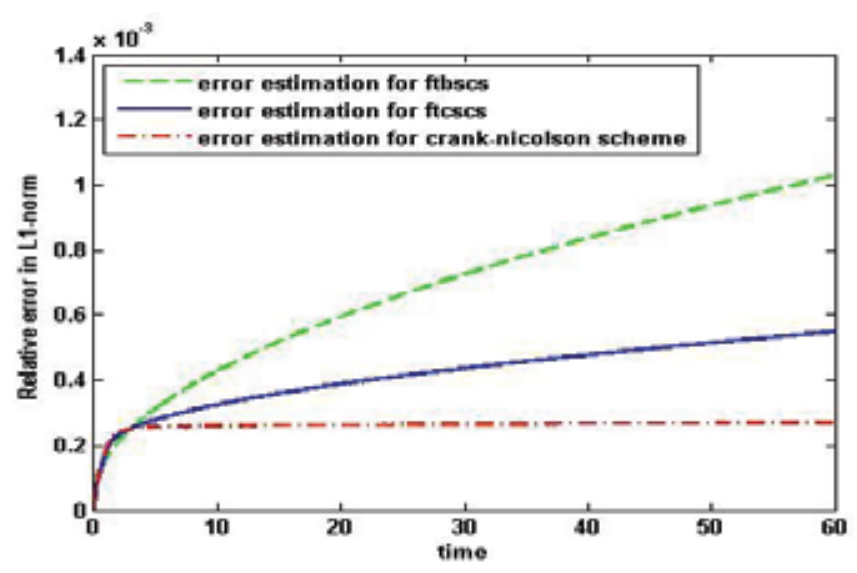

Fig. 4. Comparison of relative error for FTBSCS, FTCSCS and CNS

Figure 1 to 3 , we present concentration distribution by using FTBSCS, FTCSCS and CNS for $c=0.3$ and $D=.005$ upto time $t=60$ second in temporal grid size $\Delta t=0.06$ in spatial domain $[0,50]$ with spatial grid size $\Delta x=0.1$. Figure 4shows the comparison of relative error for three finite difference scheme. The relative error for FTBSCS below 0.0011, FTCSCS remains below 0.0005 and CNS remains below 
0.00023. From this figure, we notice that Crank-Nicolson scheme provides more accurate results than the FTBSCS and FTCSCS scheme.

\section{Convergence of relative error}

The convergence of relative error by the scheme FTBSCS, FTCSCS and CNS are shown in here. The error for different temporal and spatial step sizes are computed as established in the following figure 5 to 7 .

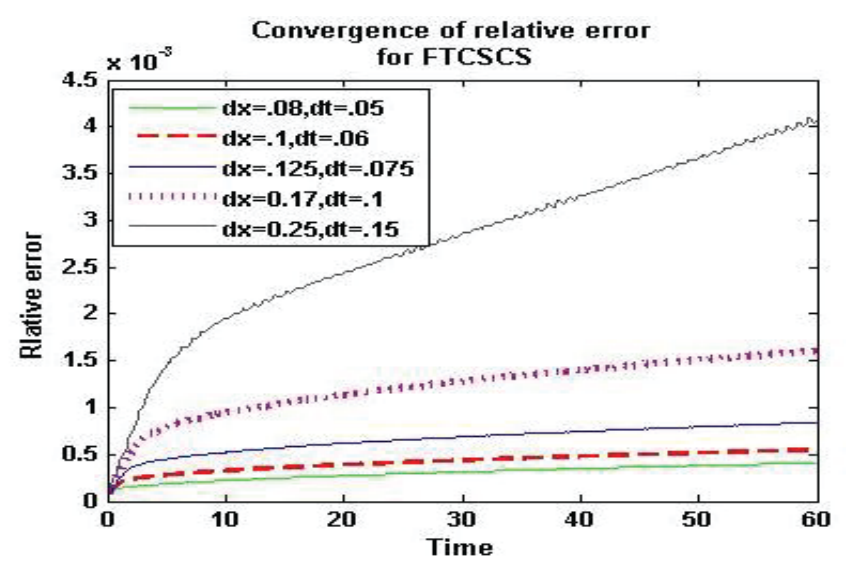

Fig. 5. Convergence of relative error for FTBSCS

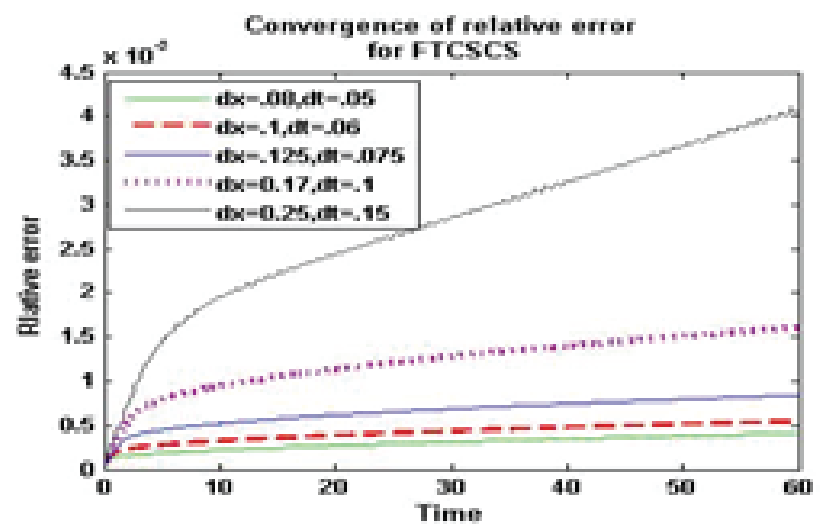

Fig. 6. Convergence of relative error for FTCSCS

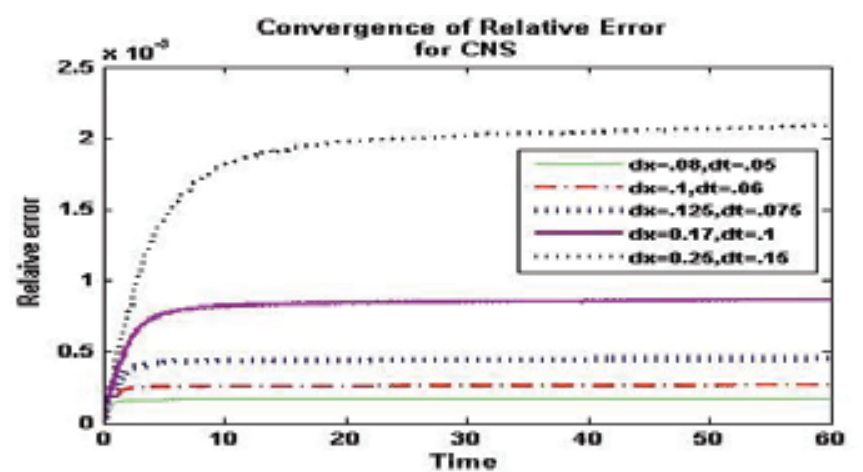

Fig. 7. Convergence of relative error for $\mathrm{CNS}$
We observe that error reduces for smaller $\Delta t$ and $\Delta x$ and FTCSCS and CNS shows goodrate of convergence. We therefore apply these two schemes for the estimation of river pollutants.

\section{Estimation of river pollution}

In this section, we present numerical simulation results for pollutant transportation with time increasing by FTCSCS. The following figure 7 shows how the pollutant concentration dispersed in a river with increase in time. River pollution occurs when pollutants are discharged directly into water bodies without treating it first.

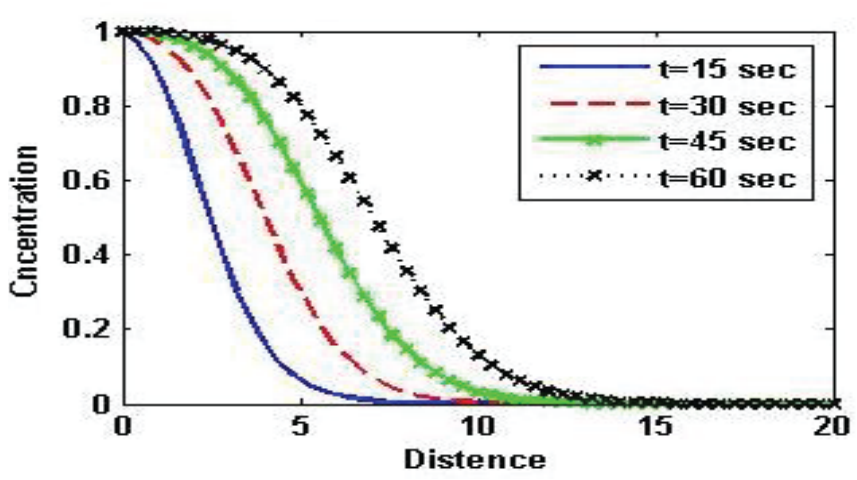

Fig. 7. Concentration distribution at different time for $u=0.1$ and $D=0.05$

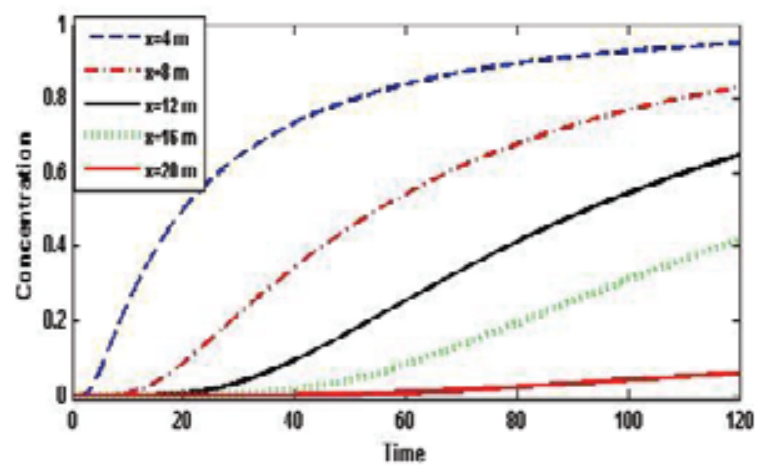

Fig. 8. Concentration distribution at different time for $u=0.1$ and $D=0.05$

Figure 7 shows the curve marked by "solid line" shows the concentration profile for 15 second, "dash line" represents the concentration profile for 30 second "the solid cross line" shows the concentration profile for 45 second, and "dot cross line" represents theconcentration profile for 60 second. We have seen that the pollutant concentration is increasing with respect to time.

Figure 8 shows the curve identified by "dash line" shows the concentration profile for $x=4 m$ and "dash dot line" represents 
the concentration profile for $x=8 m$ "solid line" shows the concentration profile for $x=12 m$ and the "dot line" curved represents the concentration profile for $x=16 \mathrm{~m}$, "last solid line" shows the concentration for $x=20 \mathrm{~m}$. Finally, we say that the pollutant concentration is increased in a still position with respect to time.

We consider zero boundary conditions at both the boundaries for Crank Nicolson scheme.
The following figure 9 to 11 shows that the river pollution are spreading with varying the diffusion term and advection term with respect to time and space for zero boundaries. We notice that no new pollution is being added. We estimate river pollution by Crank-Nicolson scheme for other boundaries and we leave it for future implementation.

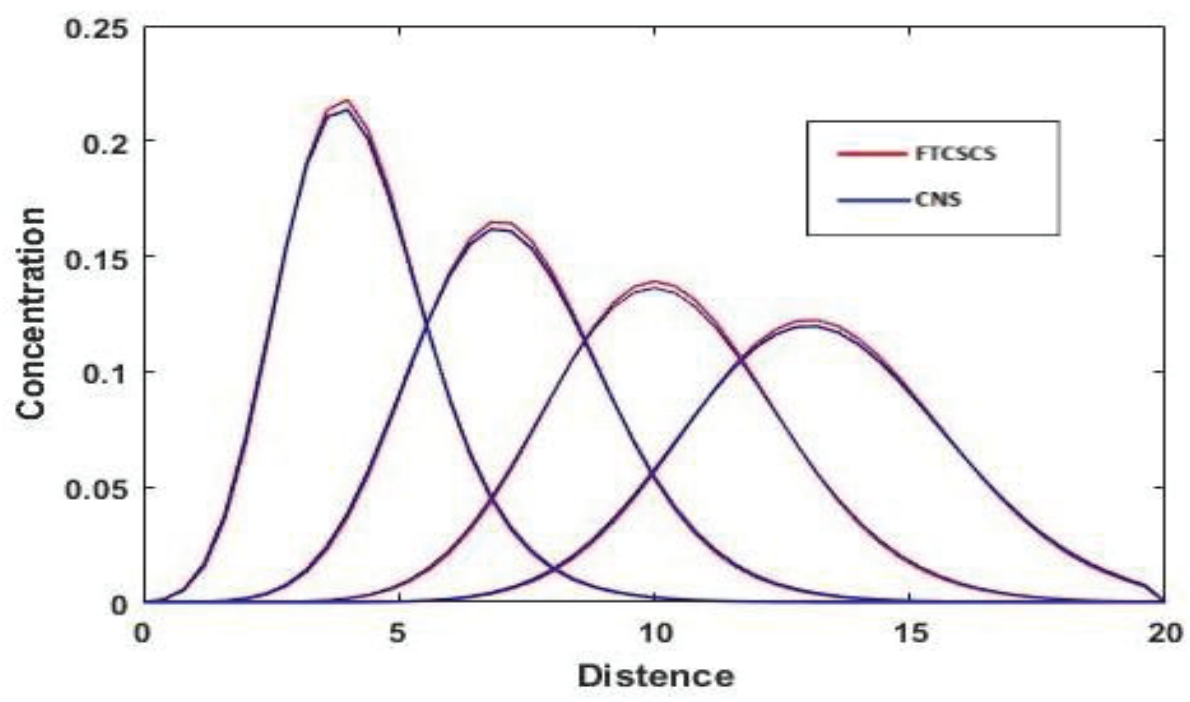

Fig. 9. Concentration distribution of FTCSCS and CNS at different time

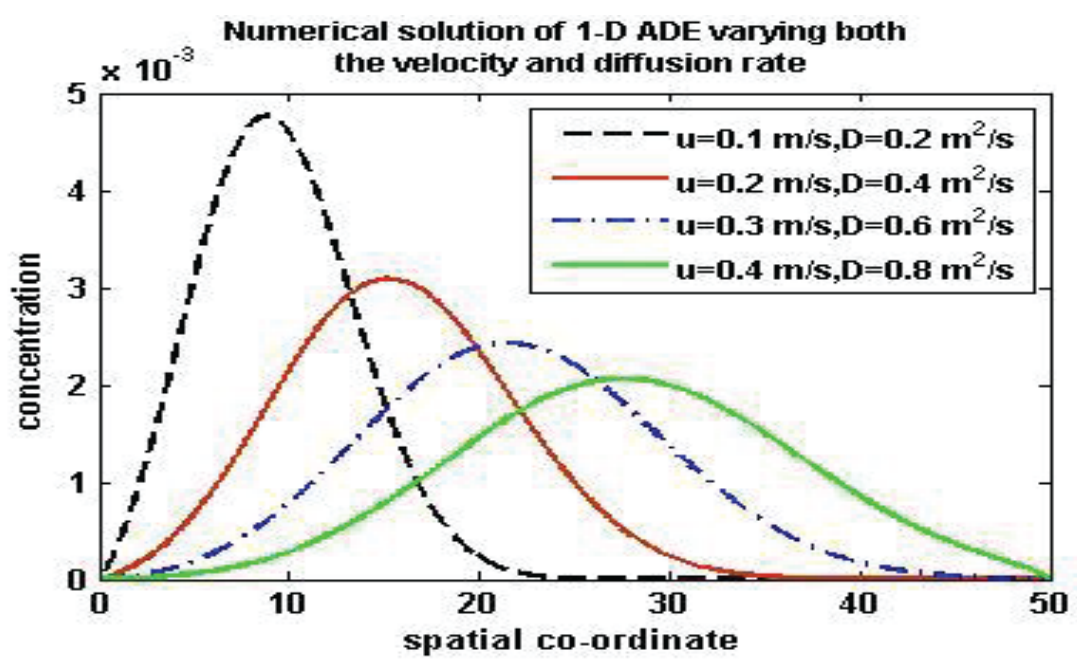

Fig. 10. Concentration distribution by CNS at different diffusion rate and fixed velocity 


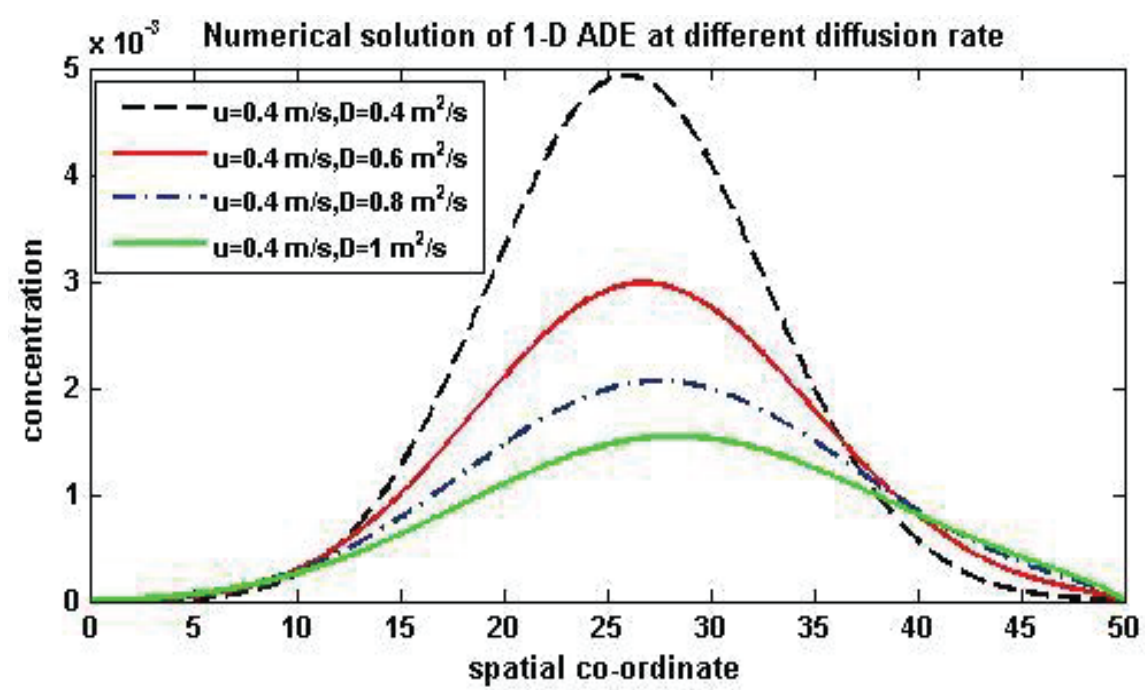

Fig. 11. Concentration distribution of CNS varying both the velocity and diffusion coefficient

\section{Conclusion}

In this paper numerical solution of advection-diffusion equation have been presented by finite difference scheme. The stability analysis for the three difference scheme FTBSCS, FTCSCS and CNS is presented. We present the error estimation graphically which shows that CNS produces more accurate results. Nevertheless FTCSCS and CNS shows good rate of convergence. These two schemes areapplied to demonstrate the pollutant distribution in a river for different time and space co-ordinates.

\section{References}

Agusto FB and Bamingbola OM (2007), Numerical treatment of the Mathematical Models for Water Pollution Research, Journal of Applied Sciences 2(5): 548-556.

Changjun Z and Shuwen L (2010), A numerical simulation of River Water Pollution using Grey Differential Model. Journal of Computer 121(122): 48-51.

Crank J and Nicolson P (1947), A practical method for numerical numerical evaluation of solutions of partial differential equations of the heat conduction type, Proc. Camb. Phil. Soc. 43(1): 50-67.

Romao, Silva and Moura (2005), Error analysis in the numeri-cal solution of $3 \mathrm{D}$ convection diffusion equation by finite difference methods. Thermal technology 8: 12-17.

Tamora J and Wadham C (2002), Numerical Solution of Advection -Diffusion Equations for Radial Flow, University of Oxford.

Thongmoon M and Mckibbin R (2006), A comparison of Somenumerical methods for the advection diffusion equation, Int. Math. Sci. 10: 49-52. http://hdl.handle.net/10179/4485 